\title{
Otimização de Variáveis Radiais na Modelagem de Fontes Radioativas Planares Usando Métodos Monte Carlo não Paramétricos
}

\author{
José W. Vieira \\ ${ }^{1}$ Instituto Federal de Educação, Ciência e Tecnologia de Pernambuco, IFPE Campus Recife \\ 50740-540, Av. Prof. Luís Freire, 500, Recife, PE \\ ${ }^{2}$ Escola Politécnica de Pernambuco, EPP-UPE \\ 50750-470, Rua Benfica, 455, Recife, PE \\ ${ }^{3}$ Departamento de Energia Nuclear, DEN-UFPE \\ 50740-540, Av. Prof. Luís Freire, 1000, Recife, PE \\ E-mail: jose.wilson@ recife.ifpe.edu.br \\ Viriato Leal Neto $^{1}$ José de M. Lima Filho ${ }^{1,2} \quad$ Marco A. de O. Domingues $^{1}$ \\ E-mail: viriatoneto@recife.ifpe.edu.br_josemelo@recife.ifpe.edu.br_marcodomingues@recife.ifpe.edu.br \\ Fernando R. de A Lima ${ }^{2,3}$ \\ Centro Regional de Ciências Nucleares do Nordeste, CRCN-NE/CNEN \\ 50740-540, Av. Professor Luiz Freire, 200, Recife, PE \\ E-mail: falima@cnen.gov.br
}

\begin{abstract}
Resumo: Nos atuais modelos computacionais de exposição (MCEs) desenvolvidos pelo GDN (estudantes e pesquisadores pertencentes ao Grupo de Pesquisa em Dosimetria Numérica elou ao Grupo de Pesquisa em Dosimetria Computacional e Sistemas Embarcados, ambos cadastrados no CNPq), um simulador do corpo humano (fantoma) é acoplado ao código Monte Carlo (MC) EGSnrc para avaliações dosimétricas (dose absorvida = energia depositada/massa). Estes MCEs precisam de um algoritmo simulador da fonte emissora de radiação. Este trabalho apresenta um algoritmo MC não paramétrico de uma fonte radioativa planar, isotrópica por rotação, dependente da variável radial, da função de distribuição acumulada (FDA) do problema e do modo como o plano é definido. No algoritmo supõe-se que o fantoma está em pé no centro de um círculo de onde emergem fótons com diversas energias. Técnicas MC foram usadas para amostrar um ponto $(r, \theta)$ de partida de um dado fóton no círculo. A variável angular $\theta$ pode ser amostrada usando a FDA uniforme. O problema principal consiste em amostrar a variável $r$ de forma que o fóton atinja uma superfície cilíndrica envolvendo o fantoma. Na abordagem apresentada neste artigo, investigou-se a influência do tamanho máximo do raio do círculo $\left(r_{\max }\right)$ e do tamanho do intervalo em que ele foi dividido $(\Delta r)$ nos resultados dosimétricos obtidos usando o MCE MSTA (Mash STAnding). Este MCE, disponivel na página do DEN-UFPE, é composto por um fantoma adulto masculino em posição ortostática -o MASH - acoplado ao EGSnrc. As implementações necessárias foram adicionadas ao software MonteCarlo, desenvolvido pelos autores e atualizado a cada novo trabalho do GDN. Os resultados aqui apresentados e comentados estabelecem um novo algoritmo para uma fonte radioativa planar, que pode ser útil em problemas envolvendo a radiação natural da Terra ou em acidentes em clínicas radiológicas.
\end{abstract}

Palavras-chave: Modelo Computacional de Exposição, Fonte Radioativa Planar, Métodos Monte Carlo não Paramétricos, EGSnrc.

\section{Exposição do Problema}

Para estimar a distribuição de dose absorvida pelos órgãos e tecidos radiossensíveis de pessoas submetidas às radiações ionizantes, é necessário realizar simulações utilizando um MCE. Tais modelos são compostos, fundamentalmente, por um simulador antropomórfico (fantoma, neologismo da palavra inglesa phantom), um código MC e um algoritmo da fonte radioativa [7].

O GDN tem contribuído significativamente para o desenvolvimento e utilização de fantomas de voxels (VOlume piXELS), desde o MAX (Male Adult voXel) [5] até MASH (Male Adult meSH) [1]. Os códigos MC estão bem estabelecidos na comunidade científica e o GDN tem utilizado o sistema EGS (Electron Gamma Shower), principalmente o EGSnrc [3] para simular o transporte de fótons e elétrons através dos materiais constituintes do fantoma, as interações destes com os átomos do meio e a avaliação da energia depositada em regiões de interesse. A dose absorvida é a razão entre a energia 
depositada e a massa da região. O Grupo também tem desenvolvido algoritmos simuladores de fontes radioativas emissoras de fótons como os apresentados em Vieira [7].

Contudo, um problema motivou o GDN em diversos estudos: o algoritmo simulador de uma fonte planar. Considerando que o problema tem simetria azimutal, em 1979 Kramer [4] deduziu a FDA para a variável radial $r$, modelando o plano como um círculo de raio $r_{\max }$. Como esta função não é inversível, não foi possível obter de um gerador de números aleatórios (GNA) radiais por via direta. Em 2006, Leal Neto e colaboradores [6] apresentaram um GNA para $r$ baseado em amostragens MC por rejeição com uma função densidade de probabilidade (FDP) exponencial por sobre o perfil da FDP do problema. Em 2013, Vieira e colaboradores [8] desenvolveram um novo GNA com base em um método MC não paramétrico, onde a variável contínua $r$ foi discretizada em intervalos $\Delta r$ de $10 \mathrm{~cm}$ com $r_{\max }=5000 \mathrm{~cm}$. Os resultados dosimétricos obtidos com este novo algoritmo foram comparados com similares obtidos por Leal Neto e colaboradores [6].

Neste trabalho, o GDN conclui o estudo apresentando o GNA não paramétrico baseado em frequências da variável radial (GNA_FR). Foi investigado o comportamento das variáveis $\Delta r$ e $r_{\max }$, a partir das grandezas de saída das simulações MC: o tempo computacional e os valores estimados para a razão $E / I N A K$, onde $E$ é a dose efetiva e INAK (INcident Air Kerma) é a grandeza de normalização KERMA (Kinetic Energy Released per unit MAss) incidente no ar. Esta razão é comumente utilizada em simulações MC envolvendo fontes radioativas externas ao corpo humano. Limites anuais de $E$ são recomendados por entidades como a CNEN (Comissão Nacional de Energia Nuclear) e a ICRP (International Commission on Radiological Protection). Assim, obtendo E/INAK e medindo INAK em um tempo $t$, nas mesmas condições simuladas, pode-se avaliar a taxa de dose efetiva causada pela fonte planar. Esta taxa pode ser extrapolada para um ano e comparada com limites estabelecidos. A melhoria do algoritmo gerador de $r$ nos MCEs utilizados reflete em avaliações dosimétricas mais exatas, fundamentais para a proteção radiológica de trabalhadores e pessoas do público.

As implementações necessárias, tanto na preparação de arquivos de entrada em formatos adequados para leitura pelo EGSnrc, quanto na análise gráfica e numérica dos dados gerados nas simulações MC, foram realizadas no ambiente de desenvolvimento do Microsoft Visual Studio 2013 (MVS 2013), usando a linguagem de programação C\# e a de marcação XAML no tipo de projeto WPF Application. A Figura 1 mostra, na janela principal do software MonteCarlo [8], a localização dos itens de menu implementados especialmente para este trabalho.

Este artigo é a sexta publicação envolvendo o software MonteCarlo, que já se constitui uma das principais inovações tecnológicas completamente desenvolvida pelo GDN. A versão atual do software está disponível em http://dosimetrianumerica.org/softwares.

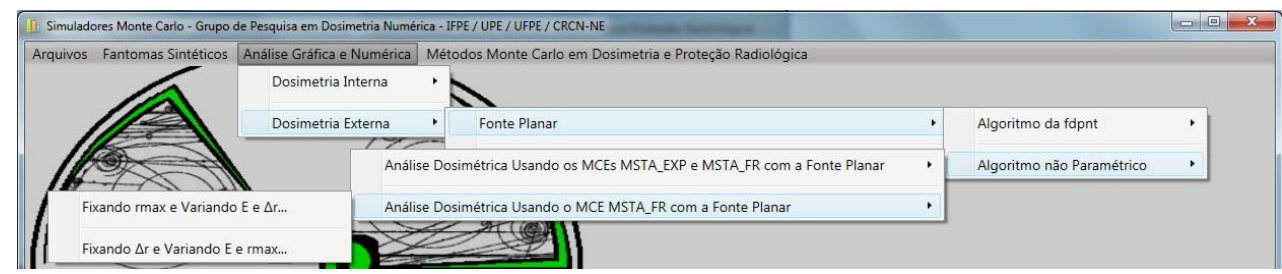

Figura 1: Janela principal do software MonteCarlo exibindo os itens para análise gráfica e numérica.

\section{Materiais e Métodos}

Este trabalho foi desenvolvido no Laboratório de Dosimetria Numérica do IFPE Campus Recife, em computadores que têm como principais itens de configuração um processador Intel ${ }^{\circledR}$ Core ${ }^{\mathrm{TM}}$ i7 CPU X990 @ 3,47GHz, 24 GB de RAM e o sistema operacional Windows 7 Ultimate de 64 bits. Nestes computadores estão instalados os softwares aqui utilizados: o EGSnrc, o MVS 2013 Ultimate para 32 bits e o MonteCarlo. O fantoma MASH foi obtido em http://www.caldose.org/, bem como os arquivos originais que compõem o MCE MSTA. A interface para análise gráfica e numérica dos resultados dosimétricos foi construída com o auxílio da versão livre da biblioteca de vínculo dinâmico AmCharts.Windows.dll (nos gráficos apresentados nos resultados há um hiperlink para a página web de onde foi obtida a versão usada). 
A Figura 2a ilustra a situação-problema modelada [6]: fótons que emergem, isotropicamente, de uma região circular e podem atingir um fantoma em pé no centro do círculo; a Figura $2 \mathrm{~b}$ mostra um esboço da geometria a ser usada para deduzir uma expressão matemática para a distribuição de fótons em uma superfície cilíndrica envolvendo o fantoma.

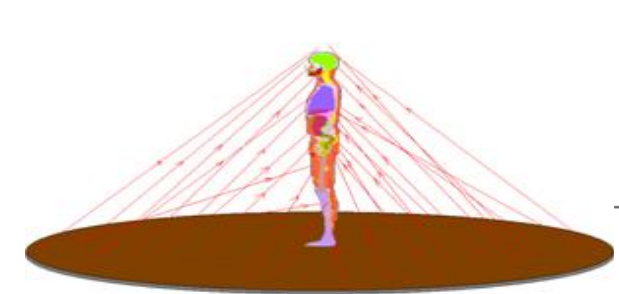

(a)

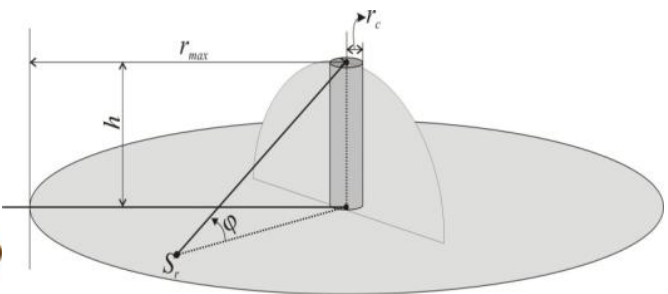

(b)

Figura 2: (a) Ilustração da situação-problema. (b) Esboço usado na formulação da fonte planar.

\section{A FDA do Problema}

A dedução da FDA radial, iniciada em Kramer [4] e concluída em Leal Neto e colaboradores [6], consiste em considerar uma área preenchida uniformemente com fontes pontuais que, isotropicamente, emitem fótons no espaço $2 \pi$ superior. Dependendo do modo de propagação do material radioativo e da constituição do meio, fontes deste tipo podem ter formas e tamanhos bem diferentes. Mas, mesmo se o tamanho de uma região circular considerada tender a infinito, por exemplo, para simular a radiação natural terrestre, é razoável supor que existe um raio limiar, $r=r_{\max }$, de tal maneira que uma pessoa em pé não seria atingida pela radiação vinda da área onde $r>r_{\max }$ de modo significativo.

Assim, o GNA pretendido deve sortear fótons emergentes de dentro de um círculo de raio $r_{\max } \mathrm{e}$ fora de um círculo de raio $r_{c}$, que atinjam a superfície cilíndrica de altura $h$ e raio $r_{c}$. Em $r<r_{c}, r$ é uniformemente amostrado. Se a distribuição dos fótons que chegam ao semicírculo de raio $h$ da Figura $2 \mathrm{~b}$ em função da distância $r$ for conhecida, pode-se, a partir do ponto $\mathrm{Sr}$ da figura, obter a FDA radial que descreve a fonte planar. Cada fóton que parte do ponto $S r$, obtido a partir de um $r$ dependente da FDA a ser deduzida e de um ângulo $\theta$, uniformemente distribuído em $[0,2 \pi]$, poderá atingir a área lateral do cilindro de raio $r_{c}$. Caso não atinja será rejeitado e novo ponto $S_{r}$ será sorteado. Mesmo os fótons que atinjam a área cilíndrica poderão ser rejeitados caso não entrem no fantoma.

A Figura 3 mostra o esquema para um fóton que parte de $S r$ sob um ângulo $\varphi(r) \in(0, \pi / 2]$. Em $\left[0, r_{c}\right]$, região sob o fantoma, $\theta=2 \pi \cdot x_{1}$ e $r=r_{c} \cdot x_{2}$, onde $x_{1}$ e $x_{2}$ são variáveis aleatórias uniformemente distribuídas em $[0,1]$ (no restante do artigo o símbolo $x$ está sempre relacionado a este tipo de variável aleatória). Resta deduzir a FDA a ser usada em $\left(r_{c}, r_{\max }\right]$.

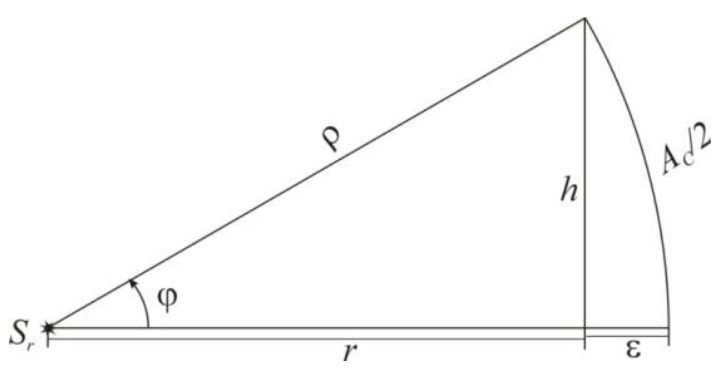

Figura 3: Variáveis usadas na dedução da fórmula da FDA do problema.

A área da calota cujo perfil da metade do seu contorno é mostrada na Figura 3 pode ser calculada usando a proporção $A_{c} / 4 \pi \rho=\varepsilon / 2 \rho$ :

$$
A_{C}=2 \pi\left(r^{2}+h^{2}\right)[1-\cos \varphi(r)] .
$$


O ângulo sólido da calota completa é $\Omega(r)=\frac{A_{C}}{r^{2}+h^{2}}=2 \pi[1-\cos \varphi(r)]$; para a meia calota, $\Omega_{m c}(r)=\pi[1-\cos \varphi(r)]$. Os fótons voam de $S r$ para o semiespaço acima do círculo sob um ângulo sólido total de $\Omega_{\text {total }}=2 \pi \mathrm{srad}$. Assim, a probabilidade $W(r)$ de um fóton sair de $S r$ e atingir a meia calota da é

$$
W(r)=\frac{\Omega_{m c}(r)}{\Omega_{\text {total }}}=\frac{1}{2}[1-\cos \varphi(r)] .
$$

O número de fótons que emerge com isotropia azimutal de um anel com largura $d r$ é

$$
d N_{i s o}=\frac{2 \pi r d r}{\pi r_{\max }^{2}} N=\frac{2 r d r}{r_{\max }^{2}} N,
$$

onde $N$ é o número total de fótons úteis simulados. Consequentemente o número de fótons que sai do anel circular no plano e chega à meia calota é, usando (2) e (3):

$$
d N=d N_{i s o} W(r)=N \frac{r}{r_{\max }^{2}}[1-\cos (\varphi(r))] d r .
$$

Assim, a FDA, que dá a probabilidade de que fótons emergentes de $\left(r_{c}, r_{\text {max }}\right]$ atinjam a meia calota da Figura 3, é dada por:

$$
F(r)=\frac{\int_{r_{c}}^{r} r^{\prime}\left[1-\cos \varphi\left(r^{\prime}\right)\right] d r^{\prime}}{\int_{r_{c}}^{r_{\max }} r[1-\cos \varphi(r)] d r}=\frac{\int_{r_{c}}^{r}\left(r^{\prime}-\frac{r^{\prime 2}}{\sqrt{r^{\prime 2}+h^{2}}}\right) d r^{\prime}}{\int_{r_{c}}^{r_{\max }}\left(r-\frac{r^{2}}{\sqrt{r^{2}+h^{2}}}\right) d r} .
$$

Com algum esforço, as integrais acima podem ser resolvidas e obtém-se:

onde

$$
F(r)=0,5 \cdot A \cdot\left[r^{2}-r \sqrt{r^{2}+h^{2}}+h^{2} \ln \left(r+\sqrt{r^{2}+h^{2}}\right)-C_{\text {min }}\right\rfloor,
$$

$$
\left\{\begin{array}{l}
C_{\text {min }}=r_{c}^{2}-r_{c} \sqrt{r_{c}^{2}+h^{2}}+h^{2} \ln \left(r_{c}+\sqrt{r_{c}^{2}+h^{2}}\right) \\
C_{\text {max }}=r_{\text {max }}^{2}-r_{\text {max }} \sqrt{r_{\text {max }}^{2}+h^{2}}+h^{2} \ln \left(r_{\text {max }}+\sqrt{r_{\text {max }}^{2}+h^{2}}\right) . \\
A=2 /\left(C_{\text {max }}-C_{\text {min }}\right)
\end{array}\right.
$$

Em Vieira e colaboradores [8], foram usados os dados da Tabela 1 como parâmetros.

\begin{tabular}{cc}
\hline Constante & Valor \\
\hline$r_{c}(\mathrm{~cm})$ & 28,80 \\
$\Delta r(\mathrm{~cm})$ & 10,00 \\
$r_{\max }(\mathrm{cm})$ & 5000,00 \\
$h(\mathrm{~cm})$ & 175,56 \\
$A\left(\mathrm{~cm}^{-2}\right)$ & $1,84408 \mathrm{E}-05$ \\
\hline
\end{tabular}

Tabela 1: Parâmetros da fonte planar [8].

\section{Porque não é Possivel Obter um GNA Direto}

Na FDA do problema, (6), $h$ é a altura do fantoma MASH e $A$ é uma constante de normalização que depende de $h$, de $r_{c}$ e de $r_{\max }$. Esta função não é inversível o que inviabiliza a amostragem de $r$ por técnicas MC diretas como demonstrado a seguir.

Para distribuições univariantes, há uma técnica de inversão generalizada que pode ser usada para obtenção de um GNA transformado a partir da FDA uniforme [2]. Supondo que $r=r(x)$ seja uma 
função contínua não decrescente de $x$, o que significaria a função $F(r)$ ? Com base na suposição acima, uma propriedade geral entre $x$ e a função $r(x)$ pode ser expressa por

$$
r(X) \leq r(x) \text { se } X \leq x \text {. }
$$

Consequentemente, têm-se as seguintes relações para as probabilidades (ou FDAs):

$$
\left\{\begin{array}{l}
P\{r(X) \leq r(x)\}=P\{X \leq x\} \Rightarrow \\
F(r)=F(x)=x
\end{array} .\right.
$$

Usando a FDA do problema:

$$
\frac{1}{C_{\max }-C_{\text {min }}}\left[r^{2}-r \sqrt{r^{2}+h^{2}}+h^{2} \ln \left(r+\sqrt{r^{2}+h^{2}}\right)-C_{\text {min }}\right]=x .
$$

Da equação (10) se vê o quanto seria complicado obter um GNA isolando $r$.

\section{O Método Monte Carlo não Paramétrico}

A versão definitiva do algoritmo simulador da fonte planar apresentada neste trabalho foi derivada diretamente da equação (6). Com valores de parâmetros similares aos da Tabela 1, podem-se calcular pontos $\left(r_{i}, F\left(r_{i}\right)\right)$ em $\left(r_{c}, r_{\max }\right]$, usando

$$
r_{i}=r_{c}+\Delta r .(i+1), i=0,1, \ldots, N
$$

e (6). Em (11), $\Delta r$ é a largura das classes na distribuição de frequências de $r$ e $N=r_{\max } / \Delta r$. Se os dados da Tabela 1 fossem usados, a região $\left(r_{c}, r_{\max }\right]$ do plano seria dividida em $N=500$ anéis concêntricos. Agora, uma amostra de $r$ pode ser obtida usando um GNA uniformes. Supondo-se que o valor $x$ foi sorteado, o algoritmo desenvolvido, usando um laço while, procura os pontos $\left(F\left(r_{1}\right), r_{1}\right)$ e $\left(F\left(r_{2}\right), r_{2}\right)$ onde $F\left(r_{1}\right) \leq x \leq F\left(r_{2}\right)$. Quando os encontra, calcula $r$ por interpolação linear:

$$
r=\left\{\begin{array}{l}
{\left[\frac{x-F\left(r_{1}\right)}{F\left(r_{2}\right)-F\left(r_{1}\right)}\right]\left(r_{2}-r_{1}\right)+r_{1}, \text { se } F\left(r_{1}\right) \neq F\left(r_{2}\right) .} \\
r_{2}, \text { caso contrário }
\end{array}\right.
$$

A equação (12) é o GNA_FR aqui apresentado. Como se vê, esta função depende apenas de um GNA uniformes, da FDA do problema e de (11) que, por sua vez, depende de $\Delta r$ e de $r_{\max }$.

\section{Resultados Obtidos e Conclusões}

Para obter dados dosimétricos que permitissem escolhas otimizadas dos valores de $\Delta r$ e $r_{\text {max }}$, foram organizados dois MCEs a partir do MSTA disponível: o MSTA_FR e o MSTA_RMAX. Ambos foram executados para $1 \mathrm{E} 7$ fótons com energias 10, 15, 20, 30, 40, 50, 60, 70, 80, 100, 150, 200, 300, $500,1000,3000,6000$ e $10000 \mathrm{keV}$. Os dados obtidos com ambos os MCEs foram adicionados ao arquivo de texto para dosimetria externa do software MonteCarlo. Como o fantoma usado é o MASH, o $E$ que aparece na razão E/INAK é a dose efetiva masculina. Para estimar o INAK na fonte planar do MCE MSTA é avaliada a fluência (número de fótons/área) através de uma hipotética esfera de raio 10 $\mathrm{cm}$ e centro a $100 \mathrm{~cm}$ do plano posicionado sobre o eixo axial do fantoma. A função de erro utilizada para a estimativa da D/INAK (D, Dose absorvida) em um dado órgão (ou tecido) na saída dos resultados é a razão $C V$ (Coeficiente de Variância) entre o desvio-padrão médio da dose absorvida nos voxels do órgão e o valor médio desta. O $C V$ para E/INAK é a média ponderada dos erros obtidos para os D/INAK, onde o peso para a radiossensibilidade de órgãos e tecidos é fornecido por entidades regulamentadoras como a ICRP. Nos resultados apresentados na sequência, $C V<5 \%$ para todos os E/INAK. O tempo computacional foi calculado no MonteCarlo a partir das strings impressas nos arquivos de saída para início e fim da simulação, que estão no formato dia-mês-ano e hora:minuto:segundo.

Nas simulações com o MCE MSTA_FR foram usados, em cm, $r_{\max }=5000$ e $\Delta r=1,2,3,4,5,7$, $10,12,15,17,20,25,50$ e 100. A Figura 4 foi obtida no software MonteCarlo fixando-se $\Delta r=5 \mathrm{~cm}$ 
na primeira coluna de gráficos e Energia $=100 \mathrm{keV}$ na segunda. Com $r_{\max }$ fixo, se espera que os valores de E/INAK mais exatos sejam os obtidos com o menor $\Delta r$ possível, já que discretizar a variável $r$ significa substituir $d r$ por $\Delta r$. Pode-se vê no gráfico da linha 1/coluna 2 da Figura 4, que os valores dosimétricos praticamente não variam com o crescimento de $\Delta r$ para a importante energia de $100 \mathrm{keV}$. Como se vê no gráfico da linha 1/coluna 1, é em torno deste valor que ocorre o máximo nas curvas $E($ ou $D) / I N A K$ x Energia do fóton. A causa deste máximo em dosimetria externa é a combinação entre o decrescimento do efeito fotoelétrico e o crescimento do efeito Compton nas interações dos fótons com os átomos dos meios à medida que a energia cresce. No gráfico da linha $2 /$ coluna 2 , se vê que o tempo computacional cai praticamente para a metade das simulações com $\Delta r=1 \mathrm{~cm}$ para as com $\Delta r=$ $5 \mathrm{~cm}$. Assim, a escolha de $\Delta r=5 \mathrm{~cm}$ não afeta as estimativas dosimétricas e melhora substancialmente o tempo computacional em relação a um intervalo de classe menor.

Nas simulações com o MCE MSTA_RMAX foram usados, em cm, $\Delta r=5$ e $r_{\max }=100,200,500$, $700,1000,2000,3000,4000,5000$ e 10000 . A Figura 5 foi obtida fixando-se $r_{\max }=10000 \mathrm{~cm}$ na primeira coluna de gráficos e Energia $=100 \mathrm{keV}$ na segunda. Com $\Delta r$ fixo e supondo-se um plano infinito, se espera que os valores de E/INAK mais exatos sejam os obtidos com o maior $r_{\max }$ possível. Pode-se vê no gráfico da linha 1/coluna 2, que os valores dosimétricos praticamente não variam a partir de $3000 \mathrm{~cm}$ para a energia de $100 \mathrm{keV}$. Assim, escolheu-se $r_{\max }=5000 \mathrm{~cm}$. Esta escolha não afeta as estimativas dosimétricas e melhora substancialmente o tempo computacional em relação ao máximo valor de $r_{\max }$ simulado, como se pode ver no gráfico da linha $2 /$ coluna 2.

Os gráficos das Figuras 4 e 5, bem como os valores de $E($ ou $D) / I N A K, C V$ e $t$ tabulados em função de 18 energias em planilhas do software MonteCarlo a partir de seleções do usuário, permitiram estabelecer os valores, $\Delta r=5 \mathrm{~cm}$ e $r_{\max }=5000 \mathrm{~cm}$. Assim, o algoritmo simulador da fonte planar acoplado ao EGSnrc a ser utilizado nos futuros trabalhos do GDN conterá estes valores.

O método não paramétrico, que consiste em utilizar pontos $(r, F(r))$ de uma FDA de onde é complicado obter um GNA analítico, pode ser adaptado para outros tipos de simulações MC. A chave para obtenção de bons estimadores de grandezas desejadas é utilizar o intervalo de classe $\Delta r$ e o limite máximo $r_{\text {max }}$ matemática, física e computacionalmente consistentes.

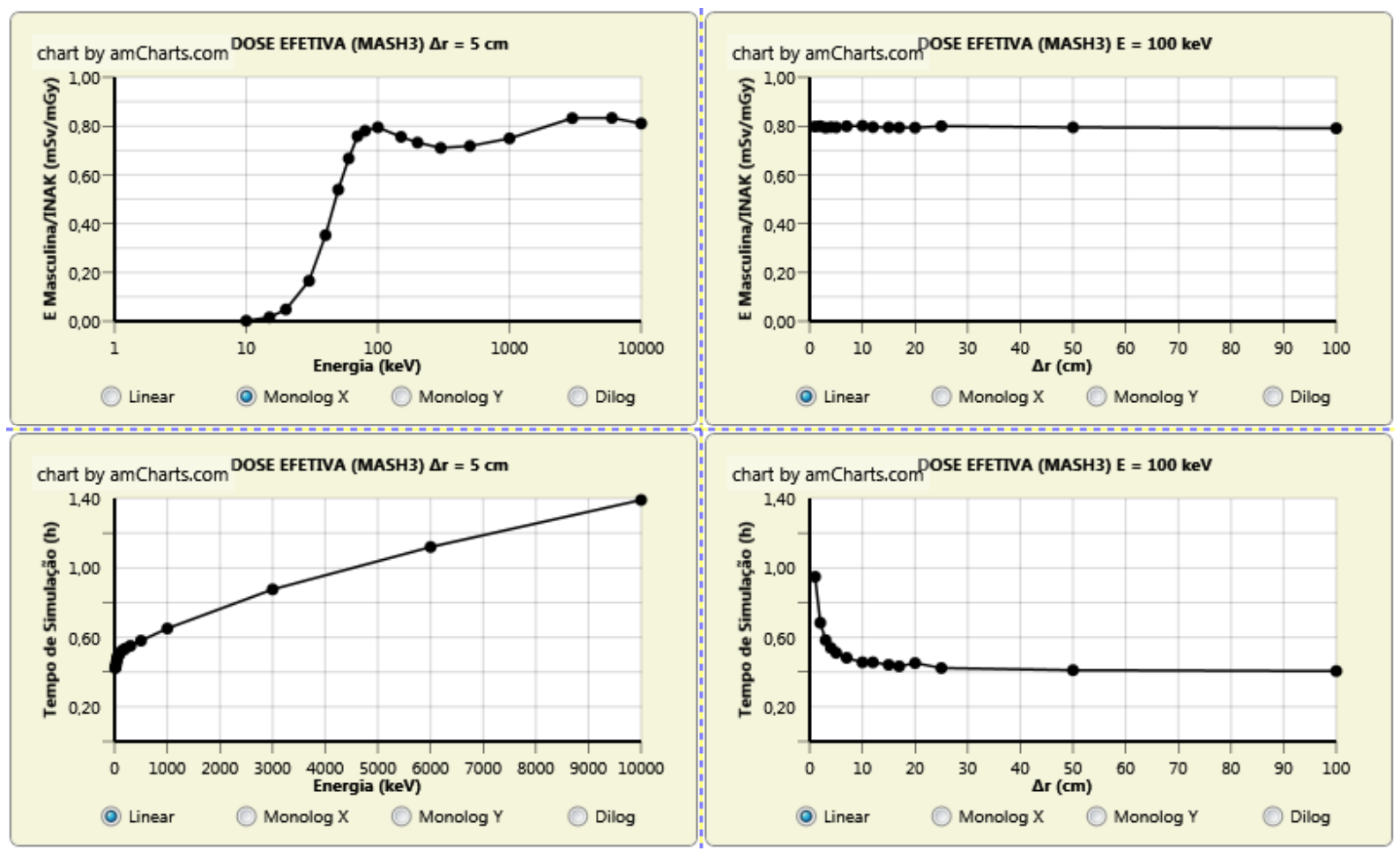

Figura 4: E/INAK e $t$ em função (coluna 1) da energia e (coluna 2) de $\Delta r$, para $r_{\max }=5000 \mathrm{~cm}$. 


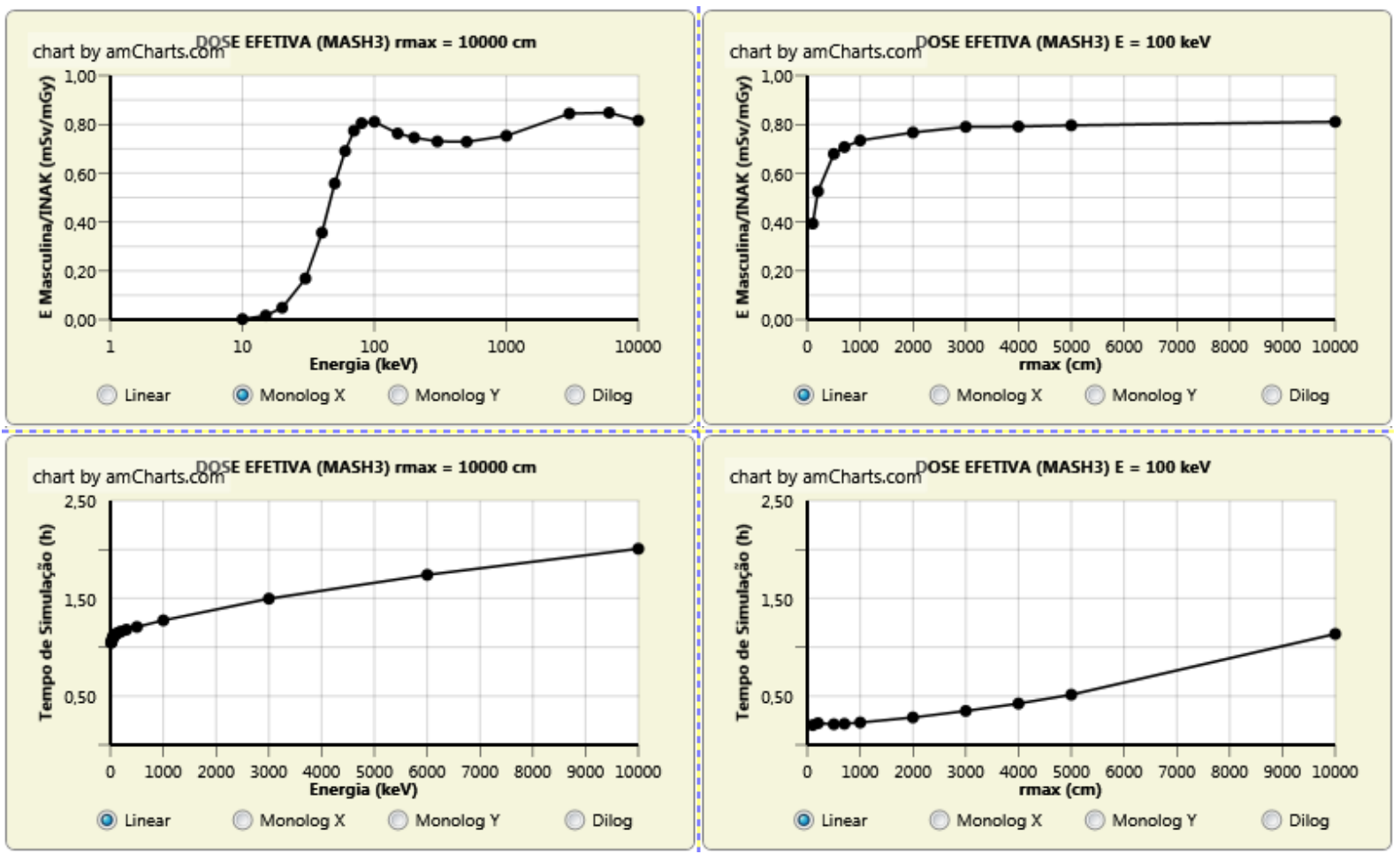

Figura 5: E/INAK e $t$ em função (coluna 1) da energia e (coluna 2) de $r_{\max }$, para $\Delta r=5 \mathrm{~cm}$.

\section{Referências}

[1] V. F. Cassola, "Desenvolvimento de Fantomas Humanos Computacionais Usando Malhas Poligonais em Função da Postura, Massa e Altura", Tese Doutorado, DEN-UFPE, 2011.

[2] M. H. Kalos; P. A. Whitlock, "Monte Carlo Methods", John Wiley \&Sons, Inc, 1986.

[3] I. Kawrakow; E. Mainegra-Hing; D. W. O. Rogers; F. Tessier; B. R. B. Walters, "The EGSnrc Code System: Monte Carlo Simulation of Electron and Photon Transport", NRCC Report PIRS701, National Research Council of Canada, Ottawa, Canada, 2013.

[4] R. Kramer, "Ermittlung von Konversionsfaktoren Zwischen Koerperdosen und Relevanten Strahlungskenngroessen bei Externer Roentgen-und Gamma-Bestrahlung", Gesellschaft fuer Strahlen-und Umweltforschung, Muenchen-Neuherberg, GSF-Bericht-S-556, 1979.

[5] R. Kramer; J. W. Vieira; H. J. Khoury; F. R. A. Lima; D. Fuelle, All About Max: A Male Adult Voxel Phantom for Monte Carlo Calculations in the Area of Radiation Protection Dosimetry, Phys. Med. Biol., 48 (2003) 1239-1262.

[6] V. Leal Neto; J. W. Veira; B. Stosic; F. R. A. Lima, Desenvolvimento Computacional de uma Fonte Radioativa Isotrópica no Solo Utilizando Técnicas Monte Carlo de Amostragem por Rejeição, "Primeiro Congresso Americano do IRPA 2006, XXIV Reunião Anual da SMSR e XVII Congresso Anual da SNM", Acapulco, México, de 3 a 8 de setembro, 2006.

[7] J. W. Vieira, "Construção de um Modelo Computacional de Exposição para Cálculos Dosimétricos Utilizando o Código Monte Carlo EGS4 e Fantomas de Voxels", Tese de Doutorado, DEN-UFPE, 2004.

[8] J. W. Vieira; V. Leal Neto; J. M. Lima Filho; F. R. A Lima, Desenvolvimento de um Método MC não Paramétrico para Simular Fontes Radioativas Planares em Modelos Computacionais de Exposição, "IV Simpósio Internacional de Inovação Tecnológica”, Aracaju, 2013. 